\title{
A role of Yueju in fast-onset antidepressant action on major depressive disorder and serum BDNF expression: a randomly double-blind, fluoxetine- adjunct, placebo-controlled, pilot clinical study
}

\author{
This article was published in the following Dove Press journal: \\ Neuropsychiatric Disease and Treatment \\ 6 August 2015 \\ Number of times this article has been viewed
}

\section{Ruyan $\mathrm{Wu}^{1, *}$ \\ Dandan Zhu',* \\ Youchun $\mathrm{Xia}^{2, *}$ \\ Haosen Wang ${ }^{2}$ \\ Weiwei Tao' \\ Wenda Xue' \\ Baomei Xia' \\ Li Ren' \\ Xin Zhou' \\ Guochun $\mathrm{Li}^{3}$ \\ Gang Chen'}

'Center for Translational Systems Biology and Neuroscience, Key Laboratory of Integrative Biomedicine of Brain Diseases, Nanjing University of Chinese Medicine, Nanjing, People's Republic of China; ${ }^{2}$ The Fourth People's Hospital of Taizhou, Taizhou, People's Republic of China; ${ }^{3}$ School of Basic Chinese Medicine, Nanjing University of Chinese Medicine, Nanjing, People's Republic of China

*These authors contributed equally to this work

\begin{abstract}
Introduction: Conventional antidepressants, including fluoxetine, have a major disadvantage in delayed onset of efficacy. Yueju, an herbal medicine used to treat mood disorders was recently found to exhibit rapid antidepressant effects. The present study was conducted to evaluate the role of Yueju in rapidly acting on major depressive disorder (MDD).

Methods: Participants were MDD patients with scores of 24-item Hamilton Depression Rating Scale (HDRS-24) $\geq 20$ and without history of antidepressant use. They randomly received daily oral doses of Yueju ( $23 \mathrm{~g}$ /day) plus fluoxetine ( $20 \mathrm{mg} /$ day) (experimental group) or placebo plus fluoxetine (control group) for 7 days. HDRS-24 was used as the primary outcome measurement at baseline, and on days 1,3,5, and 7. Concentrations of serum brain-derived neurotrophic factor (BDNF) were assessed at baseline and on days 1 and 7 .

Results: In all, 18 participants met the criteria for data analysis. Compared to baseline level, only experimental group showed significant decrease of HDRS-24 score from day 3 to day $7(P<0.05)$. Experimental group also showed significant improvement compared with control group from day 3 to day $7(P<0.05)$. No correlation between treatment outcomes with serum BDNF levels was observed. However, experimental group showed significant correlation for serum BDNF level on day 1 with day 7 ( $r=0.721, P=0.028$ ), whereas the control group did not.

Conclusion: Yueju likely contributes to fast-onset antidepressant effects on MDD. Further investigation is necessary to firmly establish the ancient formula as a safe, efficacious, and rapidly acting alternative medicine for MDD treatment.
\end{abstract}

Keywords: Yueju, major depressive disorder, HDRS-24, MADRS, rapid-onset, BDNF

\section{Introduction}

Major depressive disorder (MDD) is characteristic with the presence of loss of pleasure or interest in usually pleasurable activities (anhedonia), together with a set of other features, including anergia, changes in sleep and appetite, sadness, and suicide ideation. ${ }^{1}$ As one of the most prevalent forms of mental illness worldwide, about $10 \%-30 \%$ of women and $7 \%-15 \%$ of men likely to suffer from depression in their lifetime. ${ }^{2,3}$ Coupled with its high rate of relapsing, depression is a serious mental disorder that imposes substantial and spiritual burden on the patients as well as on public health and society. ${ }^{4,5}$ Conventional antidepressants, such as monoamine oxidase inhibitor, tricyclic antidepressants, and serotonin selective reuptake inhibitors (SSRI), take several weeks to achieve therapeutic effects. ${ }^{6}$ Additionally, almost $1 / 3$ of MDD patients have no response to the conventional medications. ${ }^{7}$ The delayed onset time and the low remission rate of conventional antidepressants
Correspondence: Gang Chen Center for Translational Systems Biology and Neuroscience, Key Laboratory of Integrative Biomedicine of Brain Diseases, Nanjing University of Chinese Medicine, 138 Xianlin Ave, Nanjing 210023, People's Republic of China

Tel $+8625858 \mathrm{I} \mid \mathrm{I} 60$

Fax +862585811160

Email chengang@njucm.edu.cn 
remain major challenges. ${ }^{8}$ Consequently, new medications that have rapid onset of antidepressant effects are urgently needed nowadays. ${ }^{6}$

Recently, ketamine, one of the $N$-methyl-D-aspartate antagonists, draws an army of attention for its high potential to ameliorate depressive symptoms within a few hours $^{9}$ and last for as long as 2 weeks. ${ }^{10}$ Ketamine rapidly increased the expression of brain-derived neurotrophic factor (BDNF) in the hippocampus. ${ }^{11}$ Serum BDNF levels were intimately associated with MDD and antidepressant responses. ${ }^{12,13}$ Serum BDNF levels are increased by ketamine in treatment-resistant MDD patients, ${ }^{14}$ in agreement with some findings that higher BDNF levels were associated with treatment with antidepressants. ${ }^{15}$ Yet, the psychotomimetic and dissociative side effects of ketamine may restrict its wide use clinically. ${ }^{16}$ Presently, a critical need remains for new treatments that act rapidly as ketamine but well-tolerated. ${ }^{16}$

Some evidence supports herbal medicine for effective and safe treatment of depression, ${ }^{17}$ for monotherapy or in adjunct with conventional antidepressants. ${ }^{18,19}$ The famous Chinese herb medicine prescription, Yueju (namely, depressionovercoming) pills, an oriental herbal formula originally prescribed 800 years ago by a pioneer Chinese medical doctor Zhu Dan-xi, is still used to treat depression, anxiety, and irritability. ${ }^{20}$ Recently, results from an experimental investigation using animals - uncovered ketamine-like rapid antidepressant effects of Yueju: acute administration of Yueju rapidly attenuated depression-like symptoms in learned helplessness paradigm ${ }^{21}$ and novelty-suppressed feeding test ${ }^{22}$ tasks which normally require chronic treatment of SSRI to exhibit antidepressant efficacy. ${ }^{23}$ A previous study showed Yueju rapidly increased hippocampal BDNF expression in mice, similar to ketamine. ${ }^{21}$ More recently, another study indicated that Gardenia jasminoides Ellis (one of the primary constituents in Yueju) also rapidly increased BDNF expression, in parallel with its rapid antidepressant efficacy in behavior paradigms. ${ }^{22}$ Based on these observations, BDNF may play a crucial role in the antidepressant mechanism of Yueju, and G. jasminoides Ellis may be responsible for such effects. Furthermore, in a chronic mild stress model that mimics clinical depression, a single administration of Yueju also showed rapid and lasting antidepressant effects similar to ketamine (Tang and Chen, unpublished observation, 2015). These findings suggest that Yueju may be potentially used as a rapid antidepressant in the clinical setting.

The aim of the present work was to assess the rapidonset efficacy of Yueju on the treatment of MDD in a 7-day, double-blinded, placebo-controlled, and randomized pilot clinical trial. Fluoxetine, a well documented late-onset SSRI, was used in adjunct with Yueju or placebo. BDNF levels in the serum before and at different times after treatment were also assessed as a putative biomarker. In the present study, 3 days of treatment with fluoxetine plus Yueju produced a modest but significant improvement, compared to unaltered clinic outcomes in patients treated with fluoxetine plus placebo. These data suggest that Yueju was crucial to induce relatively rapid antidepressant effects in MDD treatment. Additionally, the serum BDNF level on day 1 and day 7 was significantly correlated only in the experimental group, likely indicating responsiveness to the treatment.

\section{Methods}

\section{Study funding and setting}

The study was supported by Priority Academic Program Development of Jiangsu Higher Education Institutions. The study protocol was approved by the Medical Ethical Committee of the Fourth People's Hospital of Taizhou in accordance with the Declaration of Helsinki and its subsequent amendment. All participants were required to sign an informed consent document before entry into the study. Written informed consent was obtained from all the participating patients after the procedures had been fully explained.

\section{Patient selection}

Patients were recruited from referrals from outpatient psychiatric units between December 2013 and May 2014. Men and women were eligible to participate if they met the following criteria: 1) aged 18-65 years; 2) had a first-time DSM-IV diagnosis of major depression without psychotic features; ${ }^{24}$ 3) were never exposed to psychotropic drugs; and 4) had a minimum score of 20 on 24-item Hamilton Depression Rating Scale (HDRS-24).

Any of the patients with other major axis I disorders, including schizophrenia, bipolar disorders, anxiety disorders, substance-related disorders, and eating disorders, as well as the presence of any acute physical disorders, exposure to drugs known to affect either platelets or the coagulation system taken within the previous 10 days were excluded. All patients were judged clinically not to be a serious suicide risk.

A trained mental health professional who was not the treating clinician administered the ratings. High interrater interclass correlation coefficient (ICC) for the HDRS-24 (ICC $=0.92)$ and Montgomery-Asberg Depression Rating Scale (MADRS) $(\mathrm{ICC}=0.89)$ were obtained. 


\section{Randomization}

All the patients were numbered according to the order they came to the hospital. The numbers were divided into two groups using a random-numbers chart. The random numbers were sealed in opaque envelops orderly. Patients were assigned into two groups, the experimental group (Yueju plus fluoxetine) and the control group (placebo plus fluoxetine), according to the random numbers. Both patients and the depression-scale rater did not know the results of assignment.

\section{Preparation of fluoxetine}

Fluoxetine hydrochloride tablets were manufactured by Eli Lilly and Company, Indianapolis, IN, USA.

\section{Preparation of Yueju}

The medicinal plants used to prepare Yueju are Cyperus rotundus L. (Xiang Fu), Ligusticum chuanxiong Hort. (Chuan Xiong), G. jasminoides Ellis. (Zhi Zi), Atractylodes lancea (Thunb.) DC. (Cang Zu), and Massa Fermentata (Shen Qu). All the medicinal plants were purchased from Nanjing Guoyi Clinical, Medicinal Material Department (Nanjing, People's Republic of China) and authenticated by Dr Yang Lianyun, Department of Chinese Materia Medica, Nanjing University of Chinese Medicine. The herbal and placebo preparations were prepared by Chinese Medicinal Preparation Department at Jiangsu Province Hospital of Traditional Chinese Medicine, Nanjing, People's Republic of China. The crude herbal materials were processed as described in Pharmacopoeia of the People's Republic of China, 2005 Edition..$^{25}$ No additives were added except water which facilitated the formation of pills. The placebo pills were prepared to be identical to the herbal pills in shape, size, and color. To minimize the effects of distinctive smell of herbal preparations on double-blinding, both placebo and herbal tablets were contained in blister packs consisting of plastic films and aluminum foils. There were $23 \mathrm{~g}$ pills of Yueju or placebo in a blister pack for a daily oral supply. Based on the previous dose-effect relationship on Yueju in preclinical studies and Chinese medicine clinical outcome observations, ${ }^{21}$ the amount of $23 \mathrm{~g}$ pills was chosen as the testing dose in this pilot study. The dosage of fluoxetine was $20 \mathrm{mg} /$ day, a relatively safe and efficient dose. ${ }^{26}$

\section{Study design}

Patients were treated for 7 days; they were randomly assigned using a random-numbers chart to two groups, the experimental group (Yueju plus fluoxetine) and the control group (placebo plus fluoxetine). All the drugs were orally taken. The fluoxetine dose was $20 \mathrm{mg} /$ day, while the Yueju and placebo dose were $23 \mathrm{~g}$ /day. No individual or group therapy was permitted during the trial. Blood extraction was conducted at baseline, 1 st and 7 th day of the treatment to test the variance of serum concentration of BDNF.

\section{Outcome measures}

The therapeutic efficacy was evaluated at baseline, 1st, 3rd, 5th, and 7th day of the treatment by scoring according to HDRS-24 and MADRS, and the adverse reaction was scaled by Treatment Emergent Symptoms Scale. Clinical remission was defined as an achievement of a score of $\leq 8$ on HDRS-24 Clinical response was defined as a $30 \%$ decrease in HDRS-24 score from the baseline for this 7-day drug administration regimen.

\section{Blood samples}

Blood samples were drawn from the antecubital vein of MDD patients and collected into coagulation-promoting tubes. Blood extractions were performed between 9 and $10 \mathrm{am}$ to minimize the effects of possible circadian rhythm alterations. Patients had not been exposed to drugs known to affect the coagulation system within 10 days prior to blood extraction.

\section{Laboratory methods}

After obtaining the blood, the samples were centrifuged $(1,000 \times g$ for 15 minutes) immediately and serum samples were collected and stored at $-80^{\circ} \mathrm{C}$ until assayed. BDNF was measured using an anti-BDNF sandwich ELISA kit (R\&D Systems, Inc., Minneapolis, MN, USA) according to the manufacturer's instructions. Serum was diluted 1:20 with sample buffer and carried out in duplicate blind to clinical information. BDNF standard solution was diluted to concentrations from 0 to $4,000 \mathrm{pg} / \mathrm{mL}$ in a microplate reader in order to create the standard curve for BDNF levels. BDNF levels were determined by absorbance in $450 \mathrm{~nm}$ using optical density values based on the standard curve values.

\section{Statistics}

Analyses of variance with repeated measures as well as Pearson's correlations were used as appropriate. HDRS-24 score was the primary measure, and the scores obtained from other psychiatric rating scales were also tested for confirmation. Least significant difference post hoc tests examined the changes from baseline at each time point as well as group differences at each time point. The Cohen's $d$ shows the size 
of effect for the experimental-control group difference at the indicated points. Pearson's correlations were performed with the raw values. Significance was evaluated at $P<0.05$, twotailed. Data are reported as means \pm standard errors.

\section{Results}

\section{Patients}

Forty-six patients were screened, of which 20 subjects who met $D S M-I V$ criteria and first-time diagnosed with MDD were randomly assigned into experimental and placebo groups. Twenty-six patients were excluded as they did not meet inclusion criteria $(n=11)$ or refused to participate $(n=15)$ (Figure 1). Ten patients received Yueju plus fluoxetine and ten received placebo plus fluoxetine. Two patients who received placebo plus fluoxetine discontinued the study due to the intolerance of gastrointestinal symptoms and insomnia at day 1 (Figure 1) and thus data from 18 participants was analyzed.

Patients' demographic and clinical characteristics are summarized in Table 1. Overall there were eleven women and seven men. No significant difference were observed between the two groups in means \pm SEM age (experimental group: 45.70 \pm 4.62 ; control group: 47.38 $\pm 5.92, \mathrm{t}=-0.227$, $P=0.824$ ), primary HDRS-24 score (experimental group: 23.80 \pm 1.51 ; control group: $25.38 \pm 1.45$, $\mathrm{t}=-0.738, P=0.471$ ),
Table I Demographic and clinical characteristics of subjects with major depressive disorder

\begin{tabular}{lll}
\hline Characteristic & $\begin{array}{l}\text { Experimental } \\
\text { group }(\mathbf{n}=\mathbf{1 0})\end{array}$ & $\begin{array}{l}\text { Control } \\
\text { group }(\mathbf{n}=\mathbf{8})\end{array}$ \\
\hline Age (years) & $45.70 \pm 4.62$ & $47.38 \pm 5.92$ \\
HDRS-24 score & $23.80 \pm 1.51$ & $25.38 \pm 1.45$ \\
BDNF baseline $(\mathrm{ng} / \mathrm{mL})$ & $17.34 \pm 2.06$ & $15.73 \pm 1.40$ \\
\hline
\end{tabular}

Abbreviations: HDRS-24, 24-item Hamilton Depression Rating Scale; BDNF, brain-derived neurotrophic factor.

and baseline BDNF levels (experimental group: 17.34 \pm 2.06 ; control group: $15.73 \pm 1.40, \mathrm{t}=0.607, P=0.503$ ).

\section{Efficacy}

The analysis with the HDRS-24 scores showed significant effects for $\operatorname{drug}\left(F_{[1,16]}=6.363 ; P=0.023\right)$ and time $\left(F_{[1,21]}=8.290\right.$; $P=0.006)$. The interaction between drug and time did not reach significance $\left(F_{[1,21]}=2.822 ; P=0.100\right)$. Post hoc tests indicated a significant improvement for experimental group over control group from the 3rd day to the 7th day of treatment (the 3rd day: $F_{[1,6]}=7.867, P=0.013$; the 5th day: $F_{[1,16]}=7.659, P=0.014$; the 7 th day: $F_{[1,16]}=5.003, P=0.040$ ). The effect size for the drug difference was large at the $3 \mathrm{rd}$ day $(d=1.41$ [95\% confidence interval, -2.01 to 4.80$]$ ) and remained large at the 5th and 7 th days ( $d=1.39$ [95\% confidence interval, -2.29 to 5.03 ]; $d=1.13$ [95\% confidence interval, -2.96 to 5.52$]$ ). Patients in

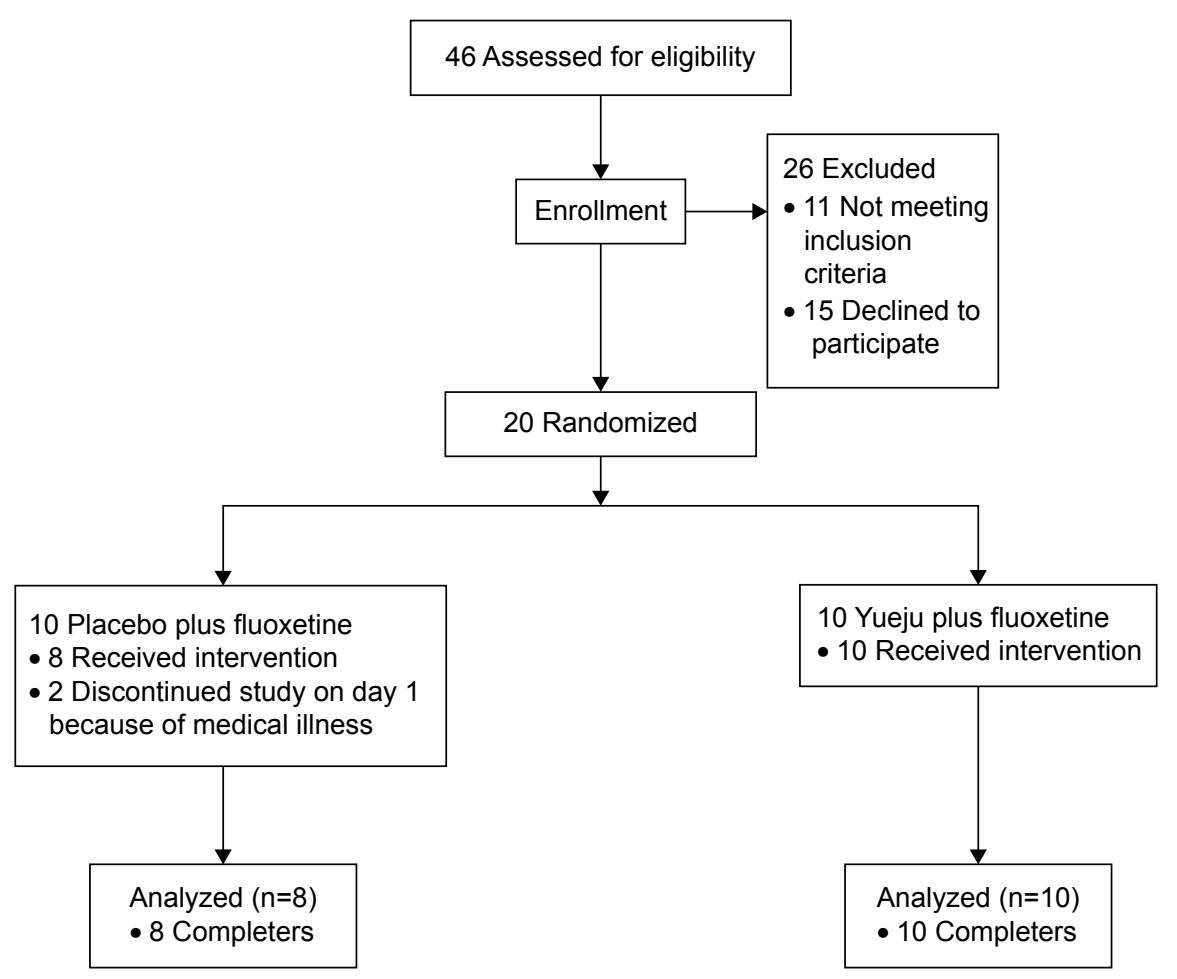

Figure I Flowchart of enrollment, randomization, discontinuation, and completion of the treatment phases $(n=18)$. 
experimental group showed a significant decrease in HDRS-24 scores $\left(F_{[4,45]}=4.637, P=0.003\right)$ over time, with all time points $P<0.05$ compared to baseline except the 1 st day, while patients in control group showed no change $\left(F_{[4,35]}=0.388, P=0.816\right)$. Table S1 and Figure 2A shows the means and standard errors for the HDRS-24 scores at each time point.

Using the data with MADRS, there was significant main effect for time $\left(F_{[1,21]}=7.791\right)$ and drug $\times$ time interactions $\left(F_{[1,21]}=4.146 ; P=0.048\right)$, but not drugs $\left(F_{[1,16]}=2.051\right.$; $P=0.171)$. In addition, there was almost a statistically significant improvement for the experimental group over the control group on 5 th day of treatment $\left(F_{[1,16]}=4.498, P=0.050\right)$. The effect size was large since the 3 rd day $(d=0.87$ [95\% confidence interval, -3.65 to 4.44$]$ ) and remained large through the study on the 5 th day $(d=1.07$ [ $95 \%$ confidence interval, -3.50 to 5.09$]$ ) and the 7 th day ( $d=0.93$ [95\% confidence interval, to 4.22-5.63]). Patients in the experimental group showed a remarkable decrease in scores of $\operatorname{MADRS}\left(F_{[4,45]}=4.077\right.$, $P=0.007$ ), with all time points $P<0.05$ compared to baseline except the 1st day, whereas patients in the control group did not show significant change overtime post 7-day treatment $\left(F_{[4,35]}=0.093, P=0.984\right)$ (Figure 2B and Table S2).

A

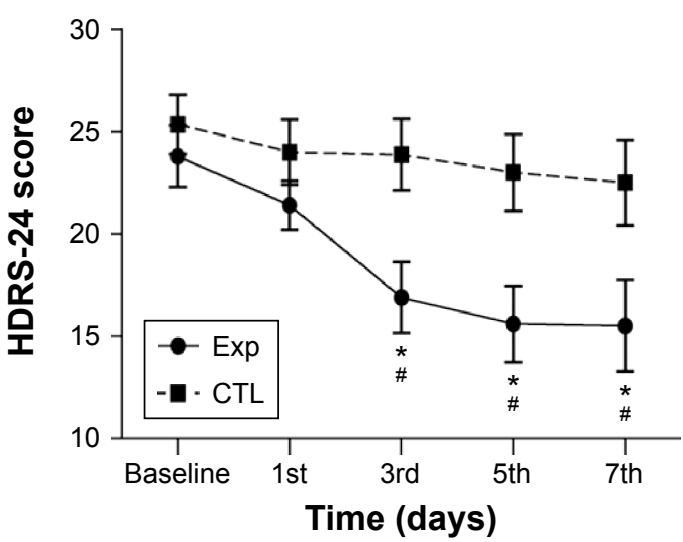

C

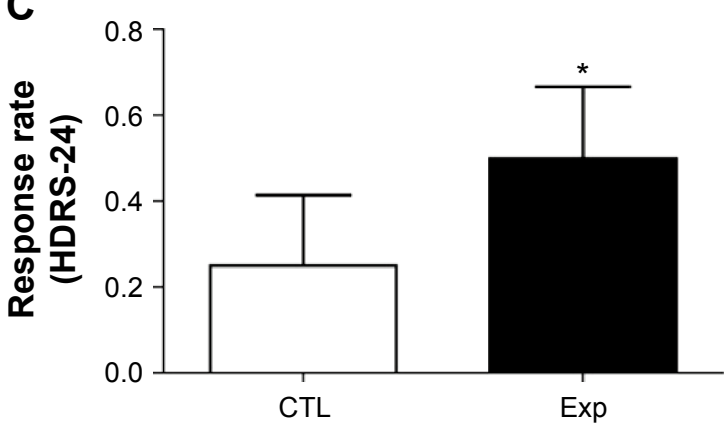

Figure $2 \mathrm{C}$ and $\mathrm{D}$ shows the response rates for HDRS-24 and MADRS throughout the study. After taking the medications for 7 days, five of ten (50\%) patients in the experimental group met response criteria using HDRS-24 data, while two of eight $(25 \%)$ patients in the control group met response criteria. There was significant difference between these two groups $(Z=-2.062, P=0.039)$. With regards to MADRS, the response rate was also different between two groups $(Z=-2.287, P=0.022)$, with five of ten $(50 \%)$ responsive patients in experimental group vs none of eight patients $(0 \%)$ in control group. No remissions were achieved in the experimental or the control groups.

\section{BDNF serum levels}

As for serum BDNF levels (Table S3), there were no significant main effects of treatment $\left(F_{[1,16]}=0.665, P=0.428\right)$, time $\left(F_{[2,32]}=2.296, P=0.119\right)$, or their interaction $\left(F_{[2,32]}\right.$ $=0.037, P=0.964)$. No correlations were found between HDRS-24 scores and BDNF serum level at baseline, day 1 or 7 post treatment. Although no significant correlations were observed between HDRS-24 scores and serum BDNF in the experimental group $(r=0.047, P=0.815)$ or control

B
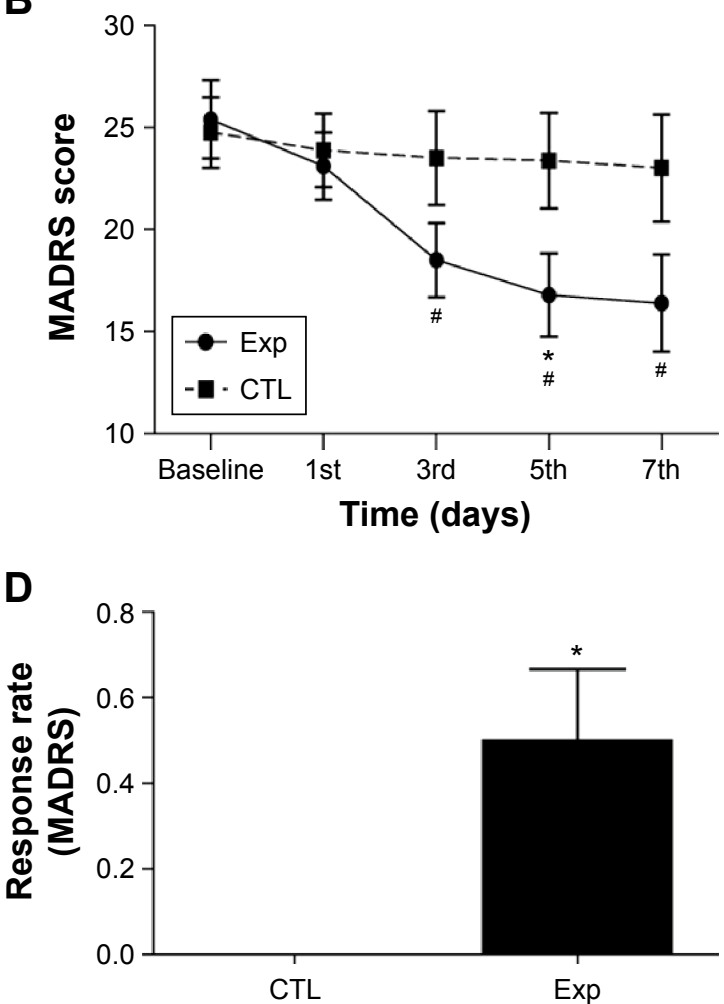

Figure 2 (A) Changes in the HDRS-24 and in (B) MADRS scores over I week post drug administration ( $\mathrm{n}=8-10)$ of experimental group (Exp, Yueju + fluoxetine) and control group (CTL, placebo + fluoxetine). Values are expressed as means and standard errors. $* P<05$, compared with control group; $\# \mathbf{P}<05$, compared with the baseline. (C) Response rate for HDRS-24 and (D) MADRS scores to experimental (Exp) and control (CTL) treatment after receiving medication for 7 days. Abbreviations: HDRS-24, 24-item Hamilton Depression Rating Scale; MADRS, Montgomery-Asberg Depression Rating Scale. 
Table 2 Correlation between baseline, the Ist day and the 7th day serum BDNF levels in experimental and control groups

\begin{tabular}{lll}
\hline & $\begin{array}{l}\text { Experimental } \\
\text { group }(\mathbf{n}=\mathbf{1 0})\end{array}$ & $\begin{array}{l}\text { Control } \\
\text { group }(\mathbf{n}=\mathbf{8})\end{array}$ \\
\hline Correlation & The 7th day & The 7th day \\
Baseline & $r=0.109, P=0.78 \mathrm{I}$ & $r=0.5 \mid 2, P=0.240$ \\
The Ist day & $r=0.721, P=0.028$ & $r=-0.104, P=0.825$ \\
\hline
\end{tabular}

Abbreviation: BDNF, brain-derived neurotrophic factor.

group ( $r=0.375, P=0.103)$, the serum BDNF level in the experimental group at day 1 and day 7 were significantly correlated ( $r=0.721, P=0.028)$, in contrast to no correlation in control group $(r=-0.104, P=0.825)$ (Table 2).

\section{Adverse effect}

There were infrequent adverse effects, mainly gastrointestinal symptoms, such as diarrhea (two for the experimental group and two for the control group) and constipation (two for the experimental group and three for the control group). Because these symptoms occurred with comparable frequency in both groups, they likely arose from the side effects of fluoxetine.

\section{Discussion}

Rapid antidepressants are urgently needed clinically. As preclinical findings indicated an herbal medicine Yueju showed ketamine-like rapid antidepressant effects, we tested whether Yueju had antidepressant potency in a relatively rapid manner clinically. In the present study, we used fluoxetine combined with Yueju or placebo to ensure patients received adequate conventional antidepressant therapy. Fluoxetine is an SSRI, which requires more than 2 weeks for observable antidepressant effects $^{27}$ and with high rates of side effects such as nausea and headaches. ${ }^{28}$ Our study focused on the first 7 days after treatment, a time when fluoxetine was incapable to elicit antidepressant effects. This was supported by the observation that in the patients receiving placebo plus fluoxetine, there were no significant changes of HDRS-24 scores over the first 7 days of treatment. In contrast, treatment with Yueju plus fluoxetine resulted in an early onset of antidepressant effects starting at 3 days and lasted for the rest of the days of testing, compared to the pretreatment baseline level, and control group. Additionally, the response rate for experimental group (Yueju plus fluoxetine) was significantly higher than the control group (placebo plus fluoxetine). The rapid antidepressant effect thus is likely owed to Yueju in the present study. To the best of our knowledge, this is the first study to demonstrate the relatively rapid antidepressant effects of an herbal medicine clinically.
The onset time of antidepressant effect of oral Yueju and fluoxetine appeared not to be as early as a single infusion of ketamine, which can exhibit antidepressant effects within hours. ${ }^{9}$ Additionally, the response rate of oral Yueju and fluoxetine was lower than ketamine infusion in the previous study: 12 (71\%) of the 17 subjects treated with ketamine met response criteria (defined as $50 \%$ reduction of the scores compared with baseline), ${ }^{6}$ whereas we found a $50 \%$ responding rate here using less stringent criteria. However, recently a study using daily oral ketamine showed a significant reduction of the depression-scale scores starting at the 14th day throughout the 28-day study. ${ }^{29}$ Thus, the onset time for a significant improvement of the mood ratings within the first few days of the treatment with fluoxetine and Yueju was still considerably quick. Although infusion of low dose of ketamine is more efficacious in quickly relieving the depressive symptoms, it can induce the side effects at the very beginning and may lead to spatial memory impairment. ${ }^{30}$ In contrast, no psychometric effects have been noticed in the present study. Therefore, Yueju plus fluoxetine may still be a promising alternative medicine to treat depression in a fast and safe manner, as well as to be used for a trial to treatmentresistant depression therapy as ketamine. ${ }^{6}$

Serum BDNF has been used as a putative maker for antidepressant responsiveness. However, different results have been reported on BDNF serum level after antidepressant treatments. Some studies failed to detect increased BDNF levels after antidepressant treatments. ${ }^{31}$ Deuschle et al reported a decline of serum BDNF level in venlafaxine-treated patients, but an increase in mirtazapine-treated patients. ${ }^{32} \mathrm{We}$ did not find a significant change of overall serum BDNF levels at 7 days in experimental or control groups, nor did we find significant correlations of serum BDNF levels with HDRS-24 scores. Observations in ketamine-infused patients showed similar results: despite a significant improvement in MADRS scores after subjects received ketamine infusion, no changes in BDNF levels were observed compared to baseline. Also, no association was found between antidepressant response and BDNF levels. ${ }^{31}$ The link between serum BDNF level and rapid antidepressant response remains to be established. Interestingly, there was a significant correlation between the 1st day and the 7th day serum BDNF levels in experimental group but not the control group. This may indicate a consistent serum BDNF level over time following Yueju plus fluoxetine treatment but not fluoxetine plus placebo treatment, suggesting responsiveness to Yueju Further clinical and preclinical studies should investigate how changes in serum BDNF level are associated with behavioral outcomes 
and whether BDNF can be used as an early indicator of the drug responsiveness.

The present study for the first time demonstrated a plausible role of an ancient herbal medicine in eliciting relatively fast antidepressant responses in MDD patients. There were, however, several limitations. First, the sample size of the present study was small. This was partially due to the relatively restrictive inclusion criteria: patients were required to be previously unexposed to psychotropic drugs, to exclude the plausible impact from the history of antidepressant administration. Also, we only included patients with relatively higher baseline HDRS-24 scores $(\geq 20)$ to test the antidepressant effects of the drugs on patients with typical symptoms of depression. Nonetheless, our preliminary results with small sample size should be interpreted with caution and prevent us from analyzing important factors influencing antidepressant responses, such as sex and estrous cycle. Second, our study is a single-center research, which may restrict generalization of our findings. Third, the study was carried out in a relatively short time, with focus on the rapid antidepressant efficacy. Future study should address the short-term and long-term therapeutic effects and with larger sample size. Fourth, although the shape and color of the placebo were similar to Yueju, the smells of Yueju and placebo were not exactly identical, which may lead to the plausible incomplete blind treatment to patients. Lastly, it would be better to include a single fluoxetine or Yueju group in order to clarify whether patients only treated with Yueju also display rapid-onset antidepressant actions. A study of monotherapy with Yueju is needed to directly test the rapid antidepressant efficacy of Yueju.

In summary, the present work suggests Yueju is potent to induce relatively rapid antidepressant effects in MDD patients with few adverse events. Further investigation with multicenter, large sample sized, randomized, long-term, and placebo-controlled clinical trials are necessary to establish the efficacy and safety of oral Yueju for the treatment of MDD.

\section{Acknowledgments}

The study was supported by Priority Academic Program Development of Jiangsu Higher Education Institutions (PAPD). We are grateful for the coordination of Bailing Su, Yingqing Sun, Aihong Wang, Zhenlin Chen, Lixia Yang, and Yan Lu from different departments of the Fourth People's Hospital of Taizhou for the study. We acknowledge Professor William Giardino from Stanford University for helping us with editing the manuscript.

\section{Disclosure}

The authors report no conflicts of interest in this work.

\section{References}

1. American Psychiatric Association. Diagnostic and Statistical Manual of Mental Disorders 5th ed. Arlington, VA: American Psychiatric Publishing; 2013.

2. Schuch JJ, Roest AM, Nolen WA, Penninx BW, de Jonge P. Gender differences in major depressive disorder: results from the Netherlands study of depression and anxiety. $J$ Affect Disord. 2014;156:156-163.

3. Briley M, Moret C. Present and future anxiolytics. I Drugs. 2000; 3(7):695-699.

4. Gibson TB, Jing Y, Smith Carls G, et al. Cost burden of treatment resistance in patients with depression. Am J Manag Care. 2010;16(5): 370-377.

5. Greden JF. The burden of disease for treatment-resistant depression. J Clin Psychiatry. 2001;62(Suppl 16):26-31.

6. Zarate CA Jr, Singh JB, Carlson PJ, et al. A randomized trial of an N-methyl-D-aspartate antagonist in treatment-resistant major depression. Arch Gen Psychiatry. 2006;63(8):856-864.

7. Trivedi MH, Rush AJ, Wisniewski SR, et al. Evaluation of outcomes with citalopram for depression using measurement-based care in STAR*D: implications for clinical practice. Am J Psychiatry. 2006;163(1):28-40.

8. Machado-Vieira R, Salvadore G, Diazgranados N, Zarate CA Jr. Ketamine and the next generation of antidepressants with a rapid onset of action. Pharmacol Ther. 2009;123(2):143-150.

9. Berman RM, Cappiello A, Anand A, et al. Antidepressant effects of ketamine in depressed patients. Biol Psychiatry. 2000;47(4):351-354.

10. Diazgranados N, Ibrahim L, Brutsche NE, et al. A randomized add-on trial of an N-methyl-D-aspartate antagonist in treatment-resistant bipolar depression. Arch Gen Psychiatry. 2010;67(8):793-802.

11. Autry AE, Adachi M, Nosyreva E, et al. NMDA receptor blockade at rest triggers rapid behavioural antidepressant responses. Nature. 2011; 475(7354):91-95.

12. Molendijk ML, Spinhoven P, Polak M, Bus BA, Penninx BW, Elzinga BM. Serum BDNF concentrations as peripheral manifestations of depression: evidence from a systematic review and meta-analyses on 179 associations (N=9,484). Mol Psychiatry. 2014;19(7):791-800.

13. Teche SP, Nuernberg GL, Sordi AO, et al. Measurement methods of BDNF levels in major depression: a qualitative systematic review of clinical trials. Psychiatr Q. 2013;84(4):485-497.

14. Haile CN, Murrough JW, Iosifescu DV, et al. Plasma brain derived neurotrophic factor (BDNF) and response to ketamine in treatment-resistant depression. Int J Neuropsychopharmacol. 2014;17(2):331-336.

15. Sen S, Duman R, Sanacora G. Serum brain-derived neurotrophic factor, depression, and antidepressant medications: meta-analyses and implications. Biol Psychiatry. 2008;64(6):527-532.

16. Zarate CA Jr, Mathews D, Ibrahim L, et al. A randomized trial of a low-trapping nonselective N-methyl-D-aspartate channel blocker in major depression. Biol Psychiatry. 2013;74(4):257-264.

17. Qin F, Wu XA, Tang Y, Huang Q, Zhang ZJ, Yuan JH. Meta-analysis of randomized controlled trials to assess the effectiveness and safety of Free and Easy Wanderer Plus, a polyherbal preparation for depressive disorders. J Psychiatr Res. 2011;45(11):1518-1524.

18. Zhang ZJ, Kang WH, Li Q, Tan QR. The beneficial effects of the herbal medicine Free and Easy Wanderer Plus (FEWP) for mood disorders: double-blind, placebo-controlled studies. J Psychiatr Res. 2007; 41(10):828-836.

19. Zhang ZJ, Kang WH, Tan QR, et al. Adjunctive herbal medicine with carbamazepine for bipolar disorders: A double-blind, randomized, placebo-controlled study. J Psychiatr Res. 2007;41(3-4):360-369.

20. Wei XH, Cheng XM, Shen JS, Wang ZT. Antidepressant effect of Yueju-Wan ethanol extract and its fractions in mice models of despair. J Ethnopharmacol. 2008;117(2):339-344. 
21. Xue W, Zhou X, Yi N, et al. Yueju pill rapidly induces antidepressantlike effects and acutely enhances BDNF expression in mouse brain. Evid Based Complement Alternat Med. 2013;2013:184367.

22. Zhang H, Xue W, Wu R, et al. Rapid antidepressant activity of ethanol extract of Gardenia jasminoides Ellis is associated with upregulation of BDNF expression in the hippocampus. Evid Based Complement Alternat Med. 2015;2015:761238.

23. Wang J, Jing L, Toledo-Salas JC, Xu L. Rapid-onset antidepressant efficacy of glutamatergic system modulators: the neural plasticity hypothesis of depression. Neurosci Bull. 2014.

24. First MB, Spitzer RL, Gibbon M, Williams AR. Structured Clinical Interview for DSM-IV TR Axis I Disorders, Research Version, Patient Edition $(S C I D-I / P)$. New York: New York State Psychiatric Institute, Biometrics Research; 2001.

25. Pharmacopoeia Commission of China, editor. Pharmacopoeia of the People's Republic of China, 2005 Edition. Beijing: Chemical Industry Press.

26. Kasper S, Fuger J, Moller HJ. Comparative efficacy of antidepressants. Drugs. 1992;43(Suppl 2):11-22; discussion 22-23.

27. Taylor MJ, Freemantle N, Geddes JR, Bhagwagar Z. Early onset of selective serotonin reuptake inhibitor antidepressant action: systematic review and meta-analysis. Arch Gen Psychiatry. 2006;63(11): 1217-1223.
28. Papakostas GI, Fava M. A metaanalysis of clinical trials comparing moclobemide with selective serotonin reuptake inhibitors for the treatment of major depressive disorder. Can J Psychiatry. 2006;51(12): 783-790.

29. Irwin SA, Iglewicz A, Nelesen RA, et al. Daily oral ketamine for the treatment of depression and anxiety in patients receiving hospice care: a 28-day open-label proof-of-concept trial. J Palliat Med. 2013;16(8): 958-965.

30. Morgan CJ, Dodds CM, Furby H, et al. Long-term heavy ketamine use is associated with spatial memory impairment and altered hippocampal activation. Front Psychiatry. 2014;5:149.

31. Machado-Vieira R, Yuan P, Brutsche N, et al. Brain-derived neurotrophic factor and initial antidepressant response to an N-methyl-Daspartate antagonist. J Clin Psychiatry. 2009;70(12):1662-1666.

32. Deuschle M, Gilles M, Scharnholz B, Lederbogen F, Lang UE, Hellweg R. Changes of serum concentrations of brain-derived neurotrophic factor (BDNF) during treatment with venlafaxine and mirtazapine: role of medication and response to treatment. Pharmacopsychiatry. 2013; 46(2):54-58. 


\section{Supplementary materials}

Table SI Key efficacy-related data based on HDRS-24

\begin{tabular}{|c|c|c|c|c|c|c|c|c|}
\hline Group & Baseline & DI & D3 & D5 & D7 & $M C$ & $\mathbf{R R}$ & $\mathbf{R e}$ \\
\hline $\operatorname{Exp}(\mathrm{ng} / \mathrm{mL})$ & $23.80 \pm 4.78$ & $21.40 \pm 3.80$ & $16.90 \pm 5.47$ & $15.60 \pm 5.87$ & $15.50 \pm 7.09$ & $8.30 \pm 2.47$ & $50 \%$ & 0 \\
\hline $\mathrm{CTL}(\mathrm{ng} / \mathrm{mL})$ & $25.37 \pm 4.10$ & $24.00 \pm 4.54$ & $23.88 \pm 4.94$ & $23.00 \pm 5.32$ & $22.50 \pm 5.90$ & $2.88 \pm 5.90$ & $25 \%$ & 0 \\
\hline
\end{tabular}

Note: All data were presented with mean \pm SD, HDRS-24 score of different time point.

Abbreviations: CTL, control; D, day; Exp, experimental; HDRS-24, 24-item Hamilton Depression Rating Scale; MC, mean change from baseline; RR, response rate; Re, remission rate; SD, standard deviation.

Table S2 Key efficacy-related data based on MADRS-24

\begin{tabular}{|c|c|c|c|c|c|c|c|c|}
\hline Group & Baseline & DI & D3 & D5 & D7 & $M C$ & $\mathbf{R R}$ & $\mathbf{R e}$ \\
\hline $\operatorname{Exp}(\mathrm{ng} / \mathrm{mL})$ & $25.40 \pm 6.08$ & $23.10 \pm 5.22$ & $18.50 \pm 5.76$ & $16.80 \pm 6.49$ & $16.40 \pm 7.58$ & $9.00 \pm 2.81$ & $50 \%$ & 0 \\
\hline CTL (ng/mL) & $24.75 \pm 4.89$ & $23.88 \pm 5.08$ & $23.50 \pm 6.52$ & $23.37 \pm 6.59$ & $23.00 \pm 7.43$ & $1.75 \pm 3.08$ & 0 & 0 \\
\hline
\end{tabular}

Note: All data were presented with mean \pm SD, MADRS-24 score of different time point.

Abbreviations: CTL, control; D, day; Exp, experimental; MADRS-24, Montgomery-Asberg Depression Rating Scale; MC, mean change from baseline; RR, response rate; $\mathrm{Re}$, remission rate; SD, standard deviation.

Table S3 Serum BDNF levels of different time points

\begin{tabular}{llll}
\hline Group & Baseline & DI & D7 \\
\hline $\operatorname{Exp}(\mathrm{ng} / \mathrm{mL})$ & $17.34 \pm 6.20$ & $16.70 \pm 3.84$ & $14.03 \pm 8.20$ \\
CTL $(\mathrm{ng} / \mathrm{mL})$ & $15.73 \pm 3.71$ & $15.61 \pm 3.45$ & $11.97 \pm 6.32$ \\
\hline
\end{tabular}

Note: All data were presented with mean $\pm S D$.

Abbreviation: CTL, control; D, day; BDNF, brain-derived neurotrophic factor; Exp, experimental; SD, standard deviation.

\section{Publish your work in this journal}

Neuropsychiatric Disease and Treatment is an international, peerreviewed journal of clinical therapeutics and pharmacology focusing on concise rapid reporting of clinical or pre-clinical studies on a range of neuropsychiatric and neurological disorders. This journal is indexed on PubMed Central, the 'PsycINFO' database and CAS, and is the official journal of The International Neuropsychiatric Association (INA). The manuscript management system is completely online and includes a very quick and fair peer-review system, which is all easy to use. Visit http://www.dovepress.com/testimonials.php to read real quotes from published authors. 